\title{
Volumetric absorptive microsampling as alternative sampling technique for renal function assessment in the paediatric population using iohexol
}

\author{
Laura Dhondt ${ }^{\mathrm{a}}$, Siska Croubels ${ }^{\mathrm{a}}$, Pieter De Cock ${ }^{\mathrm{b}, \mathrm{c}, \mathrm{d}}$, Evelyn Dhont ${ }^{\mathrm{d}}$, Siegrid De Baere ${ }^{\mathrm{a}}$, \\ Peter De Paepe ${ }^{c}$, Mathias Devreese ${ }^{a, *}$ \\ a Department of Pharmacology, Toxicology and Biochemistry, Faculty of Veterinary Medicine, Ghent University, Salisburylaan 133, Merelbeke, Belgium \\ ${ }^{\mathrm{b}}$ Department of Pharmacy, Ghent University Hospital, Corneel Heymanslaan 10, Ghent, Belgium \\ ${ }^{\mathrm{c}}$ Heymans Institute of Pharmacology, Ghent University, Corneel Heymanslaan 10, Ghent, Belgium \\ ${ }^{\mathrm{d}}$ Department of Paediatric Intensive Care, Ghent University Hospital, Corneel Heymanslaan 10, Ghent, Belgium
}

\section{A R T I C L E I N F O}

\section{Keywords:}

Iohexol

Volumetric absorptive microsampling

Liquid chromatography-tandem mass spec-

trometry

Glomerular filtration rate

Paediatrics

\begin{abstract}
A B S T R A C T
The glomerular filtration rate (GFR) is considered the best overall index for the renal function. Currently, one of the most promising exogenous markers for GFR assessment is iohexol. In this study, the suitability of volumetric absorptive microsampling (VAMS) as alternative for the conventional blood sampling and quantification of iohexol in paediatric plasma was assessed. Therefore, a new, fully validated liquid chromatography-tandem mass spectrometry (LC-MS/MS) method was developed. Subsequently, the clinical suitability was evaluated in 20 paediatric patients by comparing plasma iohexol concentrations and associated GFR values obtained by the VAMS method with those obtained by conventional blood sampling and quantification of iohexol in plasma. The developed, simple and cost-effective LC-MS/MS-method fulfilled all pre-set validation acceptance criteria. Iohexol could be accurately quantified within a haematocrit range of 20-60\% and long-term stability of iohexol in VAMS was demonstrated up to 245 days under different storage temperatures. Both iohexol plasma concentrations $(r=0.98$, mean bias: $-4.20 \%)$ and derived GFR values $(r=0.99$; mean bias: $1.31 \%)$, obtained by a conventional plasma and the VAMS method, demonstrated good correlation and acceptable bias. The agreement between the two methods was especially good for GFR values higher than $60 \mathrm{~mL} / \mathrm{min} / 1.73 \mathrm{~m}^{2}$. Nevertheless, for GFR values $<60 \mathrm{~mL} / \mathrm{min} / 1.73 \mathrm{~m}^{2}$ the accuracy compared to the plasma method was lower. However, small adjustments to the sampling protocol could probably solve this problem.
\end{abstract}

\section{Introduction}

In divergent physiological and pathological conditions, the assessment of the kidney function is essential to make optimal clinical decisions [1]. Generally, the glomerular filtration rate (GFR) is considered the best overall index of kidney function in healthy and diseased people $[2,3]$. The GFR is most frequently estimated based on serum creatinine. Nevertheless, the use of serum creatinine has some major drawbacks, since serum creatinine levels can be affected by multiple factors, making the estimated GFR (eGFR) less accurate [4]. In general, the determination of the kidney function by use of the eGFR is sufficient, but in some cases, such as chronic illness, diagnosis of early kidney disease, drug dose adjustment and monitoring the impact or toxicity of certain treatments, more accurate measurements of the renal function are needed $[1,5]$. The urinary clearance of inulin is still considered the gold standard method for GFR determination [6]. Yet, due to complexity of the protocol and high cost, intensive research has been performed to propose alternative, exogenous filtration markers that are cheap, easy to use and accurate [1]. This resulted in a diversity of filtration markers, with each having its own (dis)advantages [6].

Iohexol, a contrast agent, is proposed by Delanaye et al. as the best suited marker to replace inulin, since its properties approach those of an ideal GFR marker [7]. Iohexol is negligibly bound to plasma proteins, freely filtered by the kidneys, does not undergo tubular secretion nor reabsorption and has a low extra-renal excretion [7-11]. Consequently, urine collection is not mandatory. Furthermore, it is virtually non-toxic and carries a low cost [7]. Due to its favourable characteristics, iohexol is currently the most widely used exogenous marker for GFR measurements in Europe [7,12].

There are several liquid chromatography-based methods reported in

\footnotetext{
* Corresponding author.

E-mail address: Mathias.Devreese@ugent.be (M. Devreese).
} 
literature describing the quantification of iohexol in human plasma or serum using either ultraviolet or mass spectrometric detection [13-17]. Also other assays, including X-ray fluorescence and capillary electrophoresis, are available [18-20]. These assays generally require larger sample volumes of blood ( $>200-500 \mu \mathrm{L}$ ) obtained by phlebotomy [21]. Consequently, the last decade there has been a keen interest from the bioanalytical field in alternative microsampling techniques for quantification of iohexol [22-26]. These alternative microsampling approaches are of particular interest for sampling in the paediatric population, since they require a smaller sample volume $(\leq 50 \mu \mathrm{L})$, and are often less painful and stressful. Furthermore, they have generally fewer requirements on handling and storage $[21,27,28]$.

Currently, dried blood spot (DBS) sampling is the most frequently used dried microsampling technique for iohexol quantification [23-26]. Notwithstanding the ease of use, a major obstacle of this technique is the effect of haematocrit on the assay variability $[28,29]$. The haematocrit value of the blood can affect the resultant size of the spot, which consequently can influence the correct quantification of the analyte [28]. Luis-Lima et al. encountered possibly such a haematocrit effect when employing non-volumetric DBS for iohexol determination, resulting in inaccurate and imprecise GFR measurements [26]. To overcome the haematocrit-induced biases, the volumetric absorptive microsampling (VAMS) technique was designed to reduce the volume variation owing to alteration in haematocrit values [30]. Nevertheless, haematocrit can still influence the recovery of the analyte and its effect should therefore always be checked during validation [30]. Currently, to the authors' knowledge, only a single literature report published by Ion et al. is available describing a VAMS assay for iohexol [31].

The objective of the current study was to develop and validate an ultra-high performance liquid chromatography-mass spectrometry (UHPLC-MS/MS) assay for the quantification of iohexol using VAMS devices. In contrast to the recent paper published by Ion et al., in this paper special attention was paid to the long-term stability of the samples and the influence of haematocrit on the method accuracy [31]. Furthermore, a pilot study was designed to assess the suitability of this VAMS technology as alternative for the conventional quantification of iohexol in paediatric plasma. To the authors' knowledge, this is the first study evaluating the correlation and agreement of iohexol plasma concentrations and GFR determination obtained with VAMS and a conventional plasma method.

\section{Materials and methods}

\subsection{Chemicals, standards and reagents}

The analytical standard of iohexol, trifluoroacetic acid (TFA) and perchloric acid were purchased from Sigma-Aldrich (Bornem, Belgium). The internal standard (IS), iohexol-d5, was obtained from Alsachim (Illkirch-Graffenstaden, France). Ultrapure (UP) water was generated with a Milli-Q system (Merck-Millipore, France). Methanol (MeOH) and acetonitrile (ACN) were all of ULC-MS grade and were acquired from Biosolve (Valkenswaard, the Netherlands). Acetone, trichloroacetic acid (TCA) and formic acid (FA) were obtained from VWR International (Leuven, Belgium). Ten microliter VAMS devices (brand name Mitra ${ }^{\mathrm{TM}}$ ) were purchased from Neoteryx (Torrance, CA, USA).

For the analytical experiments, a stock solution of iohexol was prepared in UP water at a concentration of $10 \mathrm{mg} / \mathrm{mL}$. This solution was prepared in water to minimally change the nature of the blood samples when spiking. The stock solution was stored at $2-8{ }^{\circ} \mathrm{C}$. A stock solution of iohexol-d5 was made in $\mathrm{MeOH}$ at a concentration of $1 \mathrm{mg} / \mathrm{mL}$. This stock solution was aliquoted and stored at $\leq-15^{\circ} \mathrm{C}$. Working solutions were prepared by appropriate dilution of the stock solution in water, and were stored at $2-8{ }^{\circ} \mathrm{C}$.

\subsection{VAMS generation}

For method development and validation, iohexol-free whole blood was collected from the vena mediana cubiti from healthy volunteers in $\mathrm{K}_{3}$ EDTA collection tubes (Vacutest ${ }^{\circledR}$ Kima, Piove di Sacco, Italy). Calibrator and quality control (QC) samples were prepared by adding a fixed volume of working solution to fresh blank whole blood. The added volume of working solution accounted for $4 \%$ of the total sample volume. Samples were homogenized by gently inverting the samples for 20 times. Calibrators were prepared at eight different concentration levels, namely: $0.4,1.0,2.0,4.0,10,40,100$ and $400 \mu \mathrm{g} / \mathrm{mL}$. QC samples were prepared at the lower limit of quantification (LLOQ, $0.4 \mu \mathrm{g} / \mathrm{mL}$ ), low $(2.0 \mu \mathrm{g} / \mathrm{mL})$, medium $(20 \mu \mathrm{g} / \mathrm{mL})$ and high $(200 \mu \mathrm{g} / \mathrm{mL})$ level. VAMS were prepared by dipping the outer part of the absorbent VAMS tip in the blood until the tip turned completely red plus an additional $2 \mathrm{~s}$. Thereafter, VAMS were air-dried for at least $2 \mathrm{~h}$. After the drying period, VAMS samples were either immediately analysed or in case of storage stability samples, stored in zip locked bags with desiccant at room temperature, $2-8{ }^{\circ} \mathrm{C}$ or $-20{ }^{\circ} \mathrm{C}$.

\subsection{Sample preparation protocol for VAMS and plasma}

Sample preparation consisted of separating the VAMS tip from the plastic handler using a glass Pasteur pipette and transferring it into an Eppendorf cup. Subsequently, $25 \mu \mathrm{L}$ of a $50 \mu \mathrm{g} / \mathrm{mL}$ IS solution and 175 $\mu \mathrm{L}$ of extraction solvent was added. Five different types of extraction solvents were evaluated, namely $100 \% \mathrm{MeOH}, 0.01 \% \mathrm{FA}$ in $\mathrm{MeOH} / \mathrm{H}_{2} \mathrm{O}$ (80/20, v/v), 5\% perchloric acid in $\mathrm{H}_{2} \mathrm{O}(\mathrm{v} / \mathrm{v}), 10 \%$ TCA in $\mathrm{H}_{2} \mathrm{O}(\mathrm{w} / \mathrm{v})$ and $\mathrm{H}_{2} \mathrm{O} / \mathrm{ACN} /$ acetone $(30 / 35 / 35$, v/v/v). After thorough vortex mixing for $2 \mathrm{~min}$, the cups were sonicated for $30 \mathrm{~min}$, followed by an incubation at room temperature for $30 \mathrm{~min}$. Finally, the samples were centrifuged for $10 \mathrm{~min}\left(16,200 \times \mathrm{g}, 4{ }^{\circ} \mathrm{C}\right)$, whereafter the supernatant was transferred into an autosampler vial. A slightly modified protocol was used for the extraction with $\mathrm{H}_{2} \mathrm{O} / \mathrm{ACN} /$ acetone. In this case $1 \mathrm{~mL}$ of extraction solvent was added. After sonication, incubation and centrifugation, the supernatant was transferred to another tube and evaporated under a gentle $\mathrm{N}_{2}$ stream $\left(40 \pm 5{ }^{\circ} \mathrm{C}\right)$. Next, the dry residue was reconstituted in $200 \mu \mathrm{L}$ of UP water. After vortex mixing, the sample was transferred into an autosampler vial. A $5 \mu \mathrm{L}$ aliquot was injected onto the UHPLC column. For each type of extraction solvent, VAMS devices were analysed in triplicate. The final sample preparation method was selected based on a comparison of the peak areas of the analyte obtained from samples spiked at a concentration level of $100 \mu \mathrm{g} / \mathrm{mL}(\mathrm{n}=3)$ for each condition. In the final method, an injection volume of $1 \mu \mathrm{L}$ was applied.

For the determination of iohexol in plasma, blank plasma was obtained from the same healthy volunteers as used for the VAMS generation. Therefore, blood was collected in $\mathrm{K}_{3}$-EDTA collection tubes and centrifuged at $2,095 \times \mathrm{g}$ for $10 \mathrm{~min}$. The sample preparation of the plasma samples was adopted from a previously in-house developed and validated method for the determination of iohexol in human plasma. Briefly, to $100 \mu \mathrm{L}$ of plasma $12.5 \mu \mathrm{L}$ of a $50 \mu \mathrm{g} / \mathrm{mL}$ IS solution and $100 \mu \mathrm{L}$ of UP water were added. Subsequently, samples were vortex mixed for $10 \mathrm{~s}$ followed by an equilibration period of $5 \mathrm{~min}$. After addition of $15 \mu \mathrm{L}$ of trifluoroacetic acid to precipitate the proteins, samples were vortex mixed and centrifuged for $15 \mathrm{~min}\left(16,200 \times \mathrm{g}, 4^{\circ} \mathrm{C}\right)$. The clear supernatant was transferred into an autosampler vial. The injection volume was $1 \mu \mathrm{L}$.

\subsection{UHPLC-MS/MS method}

The plasma and VAMS samples were analysed using the same UHPLC-MS/MS method. Chromatographic separation was achieved on a Hypersil Gold aQ column $(1.9 \mu \mathrm{m}, 100 \times 2.1 \mathrm{~mm})$ protected by an UHPLC filter $(0.2 \mu \mathrm{m}, 2.1 \mathrm{~mm}$ ID) (ThermoFisher Scientific, Waltham, Massachusetts, United States). Gradient elution was established with a mobile phase consisting of $0.1 \%(\mathrm{v} / \mathrm{v}) \mathrm{FA}$ in $\mathrm{H}_{2} \mathrm{O}$ (eluent $\mathrm{A}$ ) and $0.1 \%(\mathrm{v} /$ 
v) $\mathrm{FA}$ in $\mathrm{MeOH}$ (eluent B) at a flow rate of $0.4 \mathrm{~mL} / \mathrm{min}$. Following gradient program was run: $0.0-1.5 \mathrm{~min}(100 \% \mathrm{~A}, 0 \% \mathrm{~B}), 1.5-2.5 \mathrm{~min}$ (linear gradient to $60 \% \mathrm{~B}), 2.5-3.5 \mathrm{~min}(40 \% \mathrm{~A}, 60 \% \mathrm{~B}), 3.5-4.0 \mathrm{~min}$ (linear gradient to $100 \%$ A), 4.0-9.0 min (100\% A, 0\% B). Column and autosampler temperature were set at $30{ }^{\circ} \mathrm{C}$ and $10{ }^{\circ} \mathrm{C}$, respectively.

The UHPLC column effluent was interfaced to a Quattro Premier XE triple quadrupole mass spectrometer (Waters, Zellik, Belgium) equipped with an electrospray ionization (ESI) probe operating in the positive ionization mode. For iohexol, two precursor-to-product ion transitions were monitored for identification $(\mathrm{m} / \mathrm{z} 822.0 \rightarrow 731.2)$ and quantification $(m / z 822.0 \rightarrow 804.0)$. For the IS, iohexol-d5, the transitions $m / z$ $827.0 \rightarrow 736.1$ and $m / z 827.0 \rightarrow 809.0$ were used for identification and quantification, respectively. A collision energy of $25 \mathrm{eV}$ for the quantification ions and $20 \mathrm{eV}$ for the identification ions was applied. The cone voltage was set at $40 \mathrm{~V}$. Masslynx software 4.1 (Waters Corp., Milford, MA, USA) was used for data acquisition and processing.

\subsection{Analytical method validation}

The VAMS method was validated by a set of parameters that were based on recommendations as defined by the European Medicines Agency (EMA) guidelines for bioanalytical method validation supplemented with recommendations defined in literature [32-36]. The validation encompassed an evaluation of the following parameters: linearity, within- and between-day accuracy and precision, lower limit of quantification (LLOQ), limit of detection (LOD), carry-over, selectivity, extraction recovery, signal suppression and enhancement (SSE), short- and long-term storage stability and haematocrit (Hct) effect.

To evaluate linearity, 8-point, matrix-matched calibration curves were constructed by plotting the ratio area analyte/area IS against the nominal concentration on three different days. Both the correlation coefficient (r) and goodness-of-fit coefficient (gof) were evaluated [33]. The latter coefficient was calculated as followed:

$g o f=\sqrt{\frac{\sum(\% \text { deviation })^{2}}{(\mathrm{n}-1)}}$

with $\backslash \%$ deviation $=$ deviation $=\frac{x_{\text {calculatedconc }}-x_{\text {nominalvalue }}}{x_{\text {nominalvalue }}} \times 100$

Values of $\mathrm{r}$ and gof should be higher than 0.99 and below $10 \%$, respectively. To determine the weighting factor (unweighted, $1 / \mathrm{x}$ or 1 / $x^{2}$ ), the gof coefficient of the 3 calibration curves are summed up per weighting factor. The weighting factor that results in the smallest sum of gof, is considered as most appropriate. In the selected model, the calculated concentrations of all included calibrator samples should comply with the criteria for accuracy.

Within-day accuracy and precision were determined using six QC samples spiked at each concentration level and analysed on the same day. Between-day accuracy and precision were evaluated by analysing two QC samples at each concentration level on 3 different days. QC samples were prepared at four concentration levels: $0.4 \mu \mathrm{g} / \mathrm{mL}$ (LLOQ), $2.0 \mu \mathrm{g} / \mathrm{mL}$ (low QC), $20 \mu \mathrm{g} / \mathrm{mL}$ (medium QC) and $200 \mu \mathrm{g} / \mathrm{mL}$ (high QC). Values for accuracy (\%bias) must be less than $\pm 15 \%$, except for the LLOQ where a bias of $\pm 20 \%$ is allowed. The precision is expressed by the relative standard deviation (RSD, \%). RSD\% values for within-day and between-day precision were considered acceptable if they were less than $\pm 20 \%$ at the LLOQ level and less than $\pm 15 \%$ at other concentration levels.

The LLOQ was the smallest measured analyte concentration which can be determined with specified degree of accuracy and precision. Moreover, the LLOQ was established as the lowest point of the calibration curve. The limit of detection, which is defined as the lowest concentration from which it is possible to deduce the presence of the analyte in the test sample with acceptable certainty, was determined by calculating the analyte concentration that corresponds with a $\mathrm{S} / \mathrm{N}$ ratio of 3 /
1, based on the $\mathrm{S} / \mathrm{N}$ ratio of the analyte in the LLOQ samples [34].

The presence of carry-over was verified by injection of neat solvent after the highest calibration sample. If a peak was observed in the elution zone of the analyte or IS, the peak area must be below $20 \%$ of the mean peak area of the LLOQ samples for the analyte and below $5 \%$ of the calibrator samples for the IS. The selectivity of the method was determined by evaluating blank VAMS samples obtained from four different adults, who did not receive iohexol. Identical criteria were applied as for carry-over.

The evaluation of SSE and extraction recovery was based on the approach suggested by Matuszewski et al. [37]. The SSE was investigated at low and high QC level in triplicate. Therefore, VAMS generated with blank blood (Hct $= \pm 40 \%$ ) were extracted by the extraction protocol (A). Thereafter, extracts were spiked at concentrations corresponding to the concentration in blood, 2 and $200 \mu \mathrm{g} / \mathrm{mL}$, respectively. Also, solvent samples, containing $10 \%$ TCA in $\mathrm{H}_{2} \mathrm{O} / \mathrm{H}_{2} \mathrm{O}(7 / 1$, v/v) were spiked at the same concentration levels (B). Absolute SSE (\%) was calculated by dividing the peak area of A by the peak area of $\mathrm{B}$, and multiplying it by 100 . To evaluate extraction recovery, samples were spiked prior (C) and post (A) extraction. Extraction recovery (\%) was determined by dividing the peak area of $\mathrm{C}$ by the peak area of $\mathrm{A}$, multiplied by 100 .

Short-term and long-term stability of iohexol in VAMS were determined after storage for 48,91 and 245 days at room temperature, $2-8{ }^{\circ} \mathrm{C}$ and $-20^{\circ} \mathrm{C}$. At each temperature and time point, triplicate QC samples were analysed at a concentration level of 2 and $200 \mu \mathrm{g} / \mathrm{mL}$. The samples stored at $-20{ }^{\circ} \mathrm{C}$ for 48 days also underwent three freeze-thaw cycles. Additionally, stability of the VAMS samples during storage for 2 days at $60{ }^{\circ} \mathrm{C}$ and stability of the processed samples in the autosampler $\left(4^{\circ} \mathrm{C}\right)$ were determined. The concentration of the stability samples was determined using a freshly prepared calibration curve. The obtained concentrations should be within $-15 \%$ to $+15 \%$ of the concentrations obtained from VAMS that were prepared together with the stability samples, but that were immediately analysed. Also the stability during sample collection and drying time was established using the approach described by Spooner et al. [35]. Whole blood was spiked at a concentration level of 2 and $200 \mu \mathrm{g} / \mathrm{mL}$. The whole blood was incubated for 30 min at $37{ }^{\circ} \mathrm{C}$, to mimic the collection of a blood sample, which was followed by leaving the blood sample at ambient room temperature for $2 \mathrm{~h}$ to mimic the drying process. The analyte to IS peak area ratio of these samples (3 replicates/concentration) was compared against those of the samples extracted immediately upon spiking. Twenty $\mu \mathrm{L}$ of whole blood was extracted as the VAMS samples. However, no sonication and incubation at room temperature was performed.

During the validation, donor blood with a haematocrit of around $40 \%$ was used. Blood with a lower/higher Hct value was prepared by adding or removing an appropriate volume of plasma to evaluate the effect of Hct on the accuracy and precision of the method. To evaluate this Hct effect, blank blood with approximate Hct levels of 20, 50 and $60 \%$ was prepared and spiked with iohexol. VAMS were generated and extracted as mentioned above. Concentrations of iohexol in these VAMS were determined using a calibration curve constructed with blood of normal Hct $( \pm 40 \%)$. Hct levels were determined by the laboratory of the Ghent University Hospital.

The iohexol plasma method was validated according to the same guidelines as the iohexol VAMS whole blood assay. For plasma analysis, matrix-matched calibrators were prepared over a concentration range of $0.5-750 \mu \mathrm{g} / \mathrm{mL}$. QC samples were prepared at the lower LLOQ $(0.5 \mu \mathrm{g} /$ $\mathrm{mL})$, low $(2.5 \mu \mathrm{g} / \mathrm{mL})$, medium $(25 \mu \mathrm{g} / \mathrm{mL})$, high $(250 \mu \mathrm{g} / \mathrm{mL})$ and upper limit of quantification $(750 \mu \mathrm{g} / \mathrm{mL}$ ) level. Additionally, the effect of the used anticoagulants on the quantification of iohexol was evaluated. Therefore, calibration curves using lithium-heparin plasma and EDTA plasma were constructed. In addition, $\mathrm{QC}$ samples ( $\mathrm{n}=3$, EDTA plasma) at low, medium and upper limit of quantification level were simultaneously analysed and quantified using the calibration curve constructed with heparin plasma. 


\subsection{Clinical suitability}

\subsubsection{Sampling and analysis}

The applicability of this validated VAMS method was determined by comparing GFR values obtained by VAMS analysis with those obtained by conventional blood sampling and quantification of iohexol in plasma. Samples were collected from 20 patients admitted to the paediatric intensive care unit (PICU). Patients received an intravenous bolus injection of iohexol (Omnipaque $300 \AA$, GE Healthcare, Eindhoven, The Netherlands) following a weight-based dosing. Children below $10 \mathrm{~kg} \mathrm{BW}$ received $1 \mathrm{~mL}$ of iohexol solution, except neonates who received $0.5 \mathrm{~mL}$. Two, 3 or $4 \mathrm{~mL}$ of Omnipaque solution was administered to children within the weight category of [10-19 kg], [20-29 kg], [30-39 kg], respectively. Above a BW of $40 \mathrm{~kg}$, a maximum of $5 \mathrm{~mL}$ iohexol solution was administered. Blood collections $(0.6-1 \mathrm{~mL} /$ collection point) were performed $20 \mathrm{~min}, 1$ and $4 \mathrm{~h}$ after iohexol administration from the arterial or venous catheter using a syringe. These sampling points were selected based on previous research performed by Dhont et al. (unpublished data). VAMS samples were prepared in duplicate by dipping the outer part of the absorbent VAMS tip in the blood at the tip of the syringe. VAMS were further handled and stored as described under section 'VAMS generation'. Furthermore, the remaining blood in the syringe was collected in lithium-heparin collection tubes. VAMS samples and plasma samples were stored for maximum 3 months at room temperature and $-80^{\circ} \mathrm{C}$, respectively.

This study was approved by the Ethics Committee of Ghent University Hospital (2018/0170). Informed consent was provided by the parents or their legal representative.

During analysis, working solutions for VAMS and plasma analyses were prepared from the same stock solution. Furthermore, the samples of each individual were analysed on the same day to avoid inter-day differences.

\subsubsection{GFR determinations}

The GFR was determined as the total body clearance of iohexol. The plasma concentration-time data was analysed by non-compartmental modelling using Phoenix ${ }^{\circledR} 8.1$ (Certara, USA). The GFR was calculated using following equation:

$C L_{I O H}=\frac{\text { Dose }}{A U C_{0 \rightarrow \text { inf }}}$

where $\mathrm{AUC}_{0 \rightarrow \text { inf }}$ is the area under the plasma concentration-time curve from time zero extrapolated to infinity, CL is the total body clearance and Dose is the dose of iohexol administered. The GFRs were indexed to body surface area (BSA) according to the formula of Dubois-Dubois [38]. Blood iohexol concentrations, obtained by VAMS ( $\mathrm{C}_{\text {IOH,VAMS }}$ ), were corrected according to patients' haematocrit to estimate the plasma levels $\left(\mathrm{C}_{\mathrm{IOH}, \mathrm{Pl}}\right)$ of iohexol using following formula:

$C_{I O H, P l}=\frac{C_{I O H, V A M S}}{1-H c t}$

The latter formula is applicable since it has previously been demonstrated that iohexol is not distributed to red blood cells $[23,39]$.

\subsubsection{Statistical analysis}

The agreement and correlation of plasma concentrations and GFRs obtained from VAMS and the conventional plasma method were assessed. The Pearson's correlation coefficient was used to evaluate the degree of correlation. Additionally, Passing-Bablok regression was performed. Bland-Altman analysis was used to further assess the agreement. Accuracy of the GFR measurements was also determined by calculating the percentage of VAMS results within 10\% (P10), 15\% (P15) and 30\% (P30) of the plasma reference method. A paired $t$-test was performed to compare plasma concentrations and GFR measurements obtained via VAMS and the conventional plasma method. Statistical analyses were performed using IBM SPSS ${ }^{\circledR}$ statistics version 26, XLSTAT version 2020.4 and Prism version 6 (GraphPad). The level of significance was set at $\alpha=0.05$. Where appropriate, mean \pm standard deviation (SD) are given.

\section{Results and discussion}

\subsection{Chromatographic separation}

The LC-MS/MS-procedure was based on a method previously published by Dhondt et al. [40]. The method was adapted in such way that the chromatographic run time was reduced from 10 to $9 \mathrm{~min}$. This was accomplished by making slight adjustments to the flow rate and mobile phase gradient. In contrast to some other published chromatographic methods, it was decided not to separate the iohexol isomers, endo- and exo-iohexol, considering no difference in clearance between the two isomers is reported in humans $[17,41,42]$. Furthermore, when iohexol analytical standard is used to prepare stock solutions, the separation of the iohexol isomers makes the method prone to errors. More specifically, when dissolving iohexol powder, first an equilibrium between the two isomers must be reached before employing it. The time to which this equilibrium is reached is mainly temperature dependent and should be tested [43]. In the final method, the retention time of iohexol and iohexol-d5 was $2.71 \mathrm{~min}$. Representative chromatograms are presented in Supplementary Fig. S1.

\subsection{VAMS extraction optimisation}

Five different extraction solvents were evaluated during this study. As depicted in Fig. 1, peak areas of iohexol were remarkably higher when extraction was performed with an aqueous solution containing $5-10 \%$ strong acid. The use of 5\% perchloric acid and 10\% TCA in $\mathrm{H}_{2} \mathrm{O}$ yielded comparable mean peak areas. This could be both the result of a higher extraction recovery, signal enhancement or the presence of signal suppression when no acid is used during extraction. The relative standard deviation (RSD\%) of the peak areas was remarkably higher for $10 \%$ TCA $(15.57 \%)$ than for $5 \%$ perchloric acid (1.80\%). This observation was related to one deviating sample in case of TCA. However, this did not seem to be the result of a lower reproducibility of the extraction, since the RSD\% of the peak area ratio of iohexol/iohexol-d5 was even better for TCA $(1.1 \%)$ than for perchloric acid (2.5\%). Based on that observation, $10 \%$ TCA was selected as final extraction procedure. Extraction recoveries with this extraction solvent were good with mean

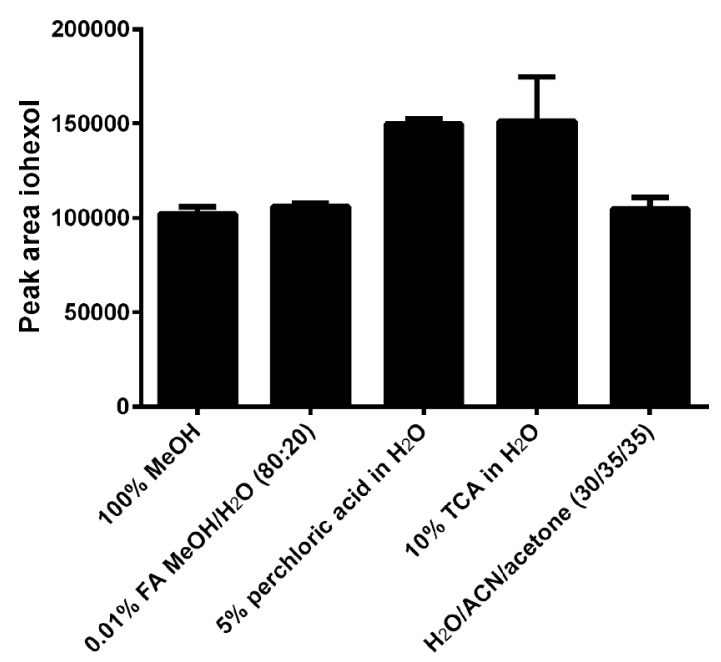

Fig. 1. Mean absolute areas ( \pm standard deviation) of iohexol obtained after extraction of VAMS samples spiked at $100 \mu \mathrm{g} / \mathrm{mL}$ with different extraction solvents. The injection volume was $5 \mu \mathrm{L}$. 
( \pm SD) values of $104( \pm 12)$ and $107( \pm 3) \%$, at low and high QC level, respectively. These extraction recoveries are higher than those obtained using an Ostro-96® well plate $(53.6-65.7 \%)$ for the extraction of iohexol in VAMS [31]. Furthermore, similar extraction recoveries with Ostro ${ }^{\circledR}$ plates were obtained for the determination of iohexol in chicken plasma [40].

\subsection{Method validation}

The calibration curves were linear within a concentration range of 0.40 and $400 \mu \mathrm{g} / \mathrm{mL}$ iohexol in blood. The best fit was obtained when applying a $1 / \mathrm{x}^{2}$ weighting. The calibration range was in the same order of magnitude as reported by Ion et al. (1-500 $\mu \mathrm{g} / \mathrm{mL})$ applying VAMS [31]. This calibration range comprises concentrations obtained after injection of the above mentioned iohexol doses to paediatric patients. Values of $r$ and gof, determined on three days, ranged from 0.997 to 0.999 and 3.70 to $7.06 \%$, respectively. The results of the within- and between-day accuracy and precision evaluation are summarized in Table 1. At all specified levels, the predefined criteria were fulfilled. The LOQ was set at $0.40 \mu \mathrm{g} / \mathrm{mL}$. The LOD was calculated to be $9.27 \mathrm{ng} / \mathrm{mL}$.

Limited carry-over was observed in the solvent sample injected after the highest calibrator. If a peak was observed at the retention time of iohexol, the area never exceeded $13.5 \%$ of the mean peak area in the LLOQ samples. Hence, the predefined criteria for carry-over were met. For the IS, no peak in the solvent samples was observed. As shown in Supplementary Fig. S1b, no interference peaks at the retention time of iohexol were observed in the blank VAMS samples. The same observations were made for iohexol-d5. Consequently, the selectivity of the method was demonstrated.

Matrix-effects, which can be observed as ion suppression or enhancement, can dramatically affect the analytical performance of an LC-MS/MS method [44]. Therefore, it is mandatory to evaluate SSE when validating such analytical methods. In this study, the mean ( \pm SD) observed absolute SSE values were $94 \pm 5 \%$ and $103 \pm 5 \%$ at low and high QC level, respectively. Hence, minimal SSE was observed. When corrected for the IS, the mean $( \pm S D)$ relative SSE values were $102 \pm 7 \%$ and $101 \pm 2 \%$ at low and high QC level. Based on these results, the use of the isotopically labelled IS, iohexol-d5, seemed not mandatory to correct for possible SSE. Nevertheless, it has previously been observed that not only endogenous compounds but also exogenous components, such as lithium heparin, can cause ion suppression or enhancement [45]. In paediatric septic patients, anti-coagulants such as heparin, are frequently administered to prevent tissue hypo-oxygenation and to attenuate organ damage and dysfunction [46]. Since the clinical samples were obtained from ICU-admitted paediatric patients, often administered various exogenous compounds such a heparin, the use of an isotopically labelled IS was preferred in order to compensate for unexpected matrix-effects.

It has previously been demonstrated that analyte recovery in VAMS can be influenced by haematocrit $[47,48]$. Generally, in healthy adults normal haematocrit values range between 39 and 50\% [49]. In children, Hct values are more variable, since it varies in function of age. At 2 months of age reported Hct levels are between 26.8 and 37.6\% (95\%

Table 1

Results of within- and between-day accuracy (\%bias) and precision (\%RSD) for QC blood VAMS samples prepared at LLOQ $(0.4 \mu \mathrm{g} / \mathrm{mL})$, low $(2.0 \mu \mathrm{g} / \mathrm{mL})$, medium $(20 \mu \mathrm{g} / \mathrm{mL})$ and high $(200 \mu \mathrm{g} / \mathrm{mL})$ iohexol concentration level.

\begin{tabular}{|c|c|c|c|c|}
\hline \multirow[t]{2}{*}{ QC level } & \multicolumn{2}{|c|}{ Within-day $(n=6)$} & \multicolumn{2}{|c|}{ Between-day $(n=3 \times 2)$} \\
\hline & $\begin{array}{l}\text { Accuracy (\% } \\
\text { bias) }\end{array}$ & $\begin{array}{l}\text { Precision (\% } \\
\text { RSD) }\end{array}$ & $\begin{array}{l}\text { Accuracy (\% } \\
\text { bias) }\end{array}$ & $\begin{array}{l}\text { Precision (\% } \\
\text { RSD) }\end{array}$ \\
\hline LLOQ & -1.9 & 9.2 & -8.4 & 9.6 \\
\hline Low & -0.1 & 5.1 & 3.5 & 5.1 \\
\hline Medium & 7.1 & 3.6 & 5.0 & 6.1 \\
\hline High & 2.4 & 4.2 & 6.2 & 2.2 \\
\hline
\end{tabular}

range) [50]. These values increase to reach adult values at an age of 10-15 years [51]. Furthermore, Hct levels can be influenced by various pathologies and medical intervention. Consequently, recovery must be determined over a wide range of Hct levels. In this study, QC samples with 3 different Hct levels $(21.0 ; 41.7 ; 63.9 \%)$ were initially prepared, to evaluate the impact of haematocrit on the accuracy and precision. These QC samples were quantified using a calibration curve prepared with blood of a normal Hct level (41.7\%). As illustrated in Fig. 2, the QC samples with a Hct level of 21.0 and $41.7 \%$ met the predefined acceptance criteria for accuracy. Nevertheless, a major deviation was observed at a Hct of $63.9 \%$. To exclude this was the result of insufficient homogenisation of the spiked blood due to high blood viscosity, analyses were repeated with samples of Hct levels of 50.1 and $60.2 \%$. These samples were evaluated using a calibration curve with a Hct level of $40.1 \%$. Special attention was given to the appropriate homogenisation of the samples. The QC samples with a Hct of $50.1 \%$ demonstrated a limited bias of $1.8 \%$ and $5.2 \%$ at low and high concentration level, respectively. In comparison to the first measurement at Hct level $\pm 60 \%$, improved accuracy (\%bias) values were obtained at second evaluation. With values of 5 and $13 \%$ at low and high QC level respectively, the predefined criterion $(\leq+15 \%)$ was met. Though there is an indication that the accuracy is somewhat lower than at the other studied Hct levels. Precision (\%RSD) results of the samples at different Hct levels were within 0.9 and $6.3 \%$, and 2.1 and $3.5 \%$ at low and high QC level, respectively. In conclusion, the quantification of iohexol in VAMS samples is guaranteed within a wide Hct-range of $21.0-60.2 \%$.

It is known that iohexol is very stable at room temperature, $-20{ }^{\circ} \mathrm{C}$ and $-80^{\circ} \mathrm{C}$ in several matrices [7]. Also in this study, iohexol demonstrated high stability. The working solutions of iohexol, stored at $2-8^{\circ} \mathrm{C}$, were considered stable over a period of minimum 254 days. The mean peak area of a working solution stored for 254 days deviated $-5.0 \%$ from the mean peak area $(n=3)$ of the working solution prepared from a fresh stock solution. Iohexol in matrix was considered stable during blood collection and drying on the VAMS. The stored samples (30 min at $37{ }^{\circ} \mathrm{C}, 2 \mathrm{~h}$ at room temperature) deviated $2.7 \%$ and $-2.8 \%$ from the immediately analysed samples at low and high QC level, respectively. As presented in Table 2, all VAMS samples stored at room temperature, $2-8{ }^{\circ} \mathrm{C}$ and $-20^{\circ} \mathrm{C}$ showed $<6 \%$ deviation after storage for up to 91 days. After storage during 245 days at $2-8{ }^{\circ} \mathrm{C}$ and $-20{ }^{\circ} \mathrm{C}$ a deviation of maximal $5.5 \%$ of the nominal values was observed. Nevertheless, after storage at room temperature during 245 days, a bias of approximately $-11 \%$ was observed at low and high QC level. This is still within the

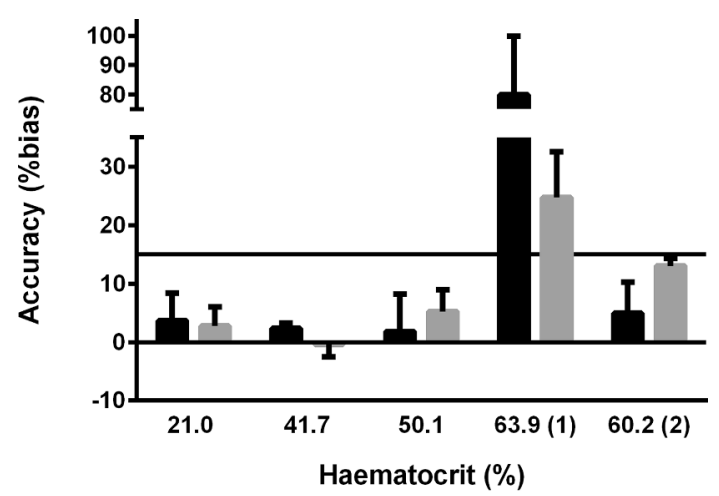

$2 \mu \mathrm{g} / \mathrm{mL} \square 200 \mu \mathrm{g} / \mathrm{mL}$

Fig. 2. Evaluation of the accuracy (\%bias) of VAMS samples ( $n=3$, low and high QC level), constructed with blood with Hct 21.0, 41.7, 50.1, 63.9 and $60.2 \%$, determined using a calibration curve constructed with blood of a Hct value of $\pm 40 \%$. Mean ( + SD) is presented. The evaluation of the Hct effect at Hct $= \pm 60 \%$ was performed in duplicate on two different days. (1) and (2) refer to experiment 1 and 2, respectively. 
Table 2

Stability of blood VAMS samples $(\mathrm{n}=3)$ spiked at low $(2 \mu \mathrm{g} / \mathrm{mL})$ and high $(200$ $\mu \mathrm{g} / \mathrm{mL})$ concentration level at different storage conditions $\left(-20{ }^{\circ} \mathrm{C}, 2-8{ }^{\circ} \mathrm{C}\right.$ and room temperature (RT)). Data are presented as \%bias against the concentration measured at time point zero (\%RSD).

\begin{tabular}{|c|c|c|c|c|c|c|}
\hline \multirow{3}{*}{$\begin{array}{l}\text { QC level } \\
\text { Storage } \\
\text { temperature }\end{array}$} & \multicolumn{6}{|c|}{$\begin{array}{l}\text { \%bias against concentration measured at time point zero (\% } \\
\text { RSD) }\end{array}$} \\
\hline & \multicolumn{3}{|l|}{ LOW } & \multicolumn{3}{|l|}{ HIGH } \\
\hline & $-20^{\circ} \mathrm{C}$ & $2-8{ }^{\circ} \mathrm{C}$ & RT & $-20^{\circ} \mathrm{C}$ & $2-8{ }^{\circ} \mathrm{C}$ & RT \\
\hline 48 days & $\begin{array}{l}-3.2^{*} \\
(8.2)\end{array}$ & $\begin{array}{l}-2.9 \\
(8.4)\end{array}$ & $\begin{array}{l}1.3 \\
(3.1)\end{array}$ & $\begin{array}{l}2.2^{*} \\
(2.6)\end{array}$ & $\begin{array}{l}-0.1 \\
(0.5)\end{array}$ & $\begin{array}{l}2.4 \\
(4.3)\end{array}$ \\
\hline 91 days & $\begin{array}{l}-4.4 \\
(9.7)\end{array}$ & $\begin{array}{l}5.7 \\
(4.9)\end{array}$ & $\begin{array}{l}-0.1 \\
(3.2)\end{array}$ & $\begin{array}{l}2.0 \\
(3.3)\end{array}$ & $\begin{array}{l}0.5 \\
(2.6)\end{array}$ & $\begin{array}{l}0.2 \\
(3.7)\end{array}$ \\
\hline 245 days & $\begin{array}{l}-4.7 \\
(1.3)\end{array}$ & $\begin{array}{l}-5.5 \\
(8.7)\end{array}$ & $\begin{array}{l}-11.0 \\
(2.0)\end{array}$ & $\begin{array}{l}0.6 \\
(2.4)\end{array}$ & $\begin{array}{l}-3.1 \\
(5.4)\end{array}$ & $\begin{array}{l}-10.6 \\
(6.9)\end{array}$ \\
\hline
\end{tabular}

* including three freeze-thaw cycles

predefined $-15-15 \%$ interval, however this can be a possible sign of degradation or a storage-induced recovery issue [52]. In the study of Salvador et al., iohexol DBS were stable at room temperature up to 9 months [24]. No significant effect of 3 freeze-thaw steps on the analyte concentration was observed. Additionally, the samples stored for 2 days at $60{ }^{\circ} \mathrm{C}$ also demonstrated acceptable bias, with values of $-5.8 \%$ and $-8.0 \%$ at low and high concentration level, respectively. The high stability of iohexol makes it possible that clearance investigations can be performed in various medical settings. After sample collection, the samples can be easily shipped, without the need for dry ice, to a central laboratory. Stability of processed samples at autosampler temperature was demonstrated for up to 7 days. The autosampler stored QC samples deviated $-3.64 \%$ (low QC) and $-0.68 \%$ (high QC) from their concentration measured immediately after preparation.

A summary of the validation results for the plasma analysis method along with the acceptance criteria can be found in supplementary Table S1. The type of anticoagulants did not affect the quantification of iohexol in plasma (Fig. S2 and Table S2).

\subsection{Clinical suitability}

Twenty patients were recruited for this study. The patient characteristics are presented in Table 3. In 7 patients, iohexol was administered a second time at $\mathbf{2 - 5}$ days after the first administration. In one patient iohexol was administered 3 times in a 9-day period. In four occasions, only 2 sampling points instead of the predefined 3 points were available for GFR determinations. Therefore, these GFR measurements were not included in the comparison of VAMS and the conventional plasma method. Eventually, 83 paired patients samples and 25 paired GFR measurements using both the conventional sampling technique and VAMS technique were obtained. With this number of samples, the minimal recommended amount of 20 paired samples was surpassed. The FDA recommends a minimum of 20 paired samples in bridging studies to appropriately evaluate the agreement between two different bioanalytical methods [53].

An acceptable correlation ( $\mathrm{r}=0.98, \mathrm{p}<0.0001)$ was observed between plasma concentrations obtained by the conventional technique

Table 3

Characteristics of the patients $(n=20)$ obtained during the first iohexol administration.

\begin{tabular}{ll}
\hline Age, years: median (range) & $1.71(0.0025-15.58)$ \\
Gender, male: $\mathrm{n}(\%)$ & $11(55 \%)$ \\
Weight, kg: median (range) & $11.5(3.1-70)$ \\
Height, cm: median (range) & $86(47-164)$ \\
BSA, m ${ }^{2}:$ median (range) & $0.54(0.19-1.73)$ \\
Haematocrit, \%: median (range) & $30.3(23.6-41.2)$ \\
Serum creatinine, mg/dL: median (range) & $0.47(0.32-22.00)$ \\
\hline
\end{tabular}

and calculated plasma concentrations from the VAMS samples. PassingBablok analysis (Fig. 3a) revealed no systematic error as zero is included in the $95 \%$ confidence interval (CI) of the intercept. A small proportional error was observed, since the $95 \%$ CI (0.91 to 0.97) of the slope did not include one. Though, Gleason et al. discussed previously that incorporation of an equivalence interval for the $95 \%$ CI could be a more appropriate approach in cases were very narrow CI of slopes (or intercepts) are observed. As an example, it was proposed that the $95 \% \mathrm{CI}$ of the slope should fall within $0.9-1.1(10 \%)$ for the methods to be considered comparable [54]. The latter interval compromises the $95 \%$ CI of the slope observed in this study. As a consequence, the iohexol VAMS and conventional method can be considered comparable. The agreement between the two methods was demonstrated by BlandAltman comparison as shown in Fig. 3b [55]. A slight negative mean bias of $-4.20 \%$ ( $95 \%$ CI -6.58 to $-1.81 \%$ ) was observed. Although limited, and therefore of little analytical and clinical relevance, this bias appeared statistically significant $(\mathrm{p}=0.001)$. To which aspect(s) this bias is attributed is unknown. This bias could be something inherent to iohexol dried blood microsampling. Though an analytical source can also not be excluded, since a bias of $-4.20 \%$ is still within the acceptance criteria of accuracy when performing bioanalytical method validation. A study on a larger scale would allow the identification of the source of the observed bias in this study. A total of $94 \%$ of the paired plasma-VAMS samples were within $20 \%$ of the mean plasma concentration obtained via plasma and VAMS analysis. In this way, the predefined FDA acceptance criteria ( $67 \%$ should be $\pm 20 \%$ of the mean) for incurred sample analyses are fulfilled [53].

As illustrated in Fig. 4a, a good correlation between the GFR estimations using both techniques was observed $(r=0.99, \mathrm{p}<0.0001)$. Passing-Bablok analysis of the GFRs indicated good overall agreement as values one and zero are included in the $95 \%$ CI of the slope (0.98-1.12) and the intercept ( -7.18 to 3.92 ), respectively. Consequently, no proportional or systematic errors were found in the comparison of GFR using both sampling methods. The agreement between GFR measurements using VAMS and conventional blood sampling and quantification for all patients $(n=25)$, patients with GFR below $60(n=8)$ and above $60 \mathrm{~mL} / \mathrm{min} / 1.73 \mathrm{~m}^{2}(\mathrm{n}=17)$ are presented in Table 4. Bland-Altman analysis for all patients demonstrated a slight mean positive bias of $1.31 \%$ (95\% CI -4.84 to $7.47 \%$ ), which was not statistically significant $(\mathrm{p}=0.10)$. Although not significant, there seems a slight tendency of overestimation of the GFR by VAMS as depicted in Fig. 4b. Bland-Altman analysis in the $>60 \mathrm{~mL} / \mathrm{min} / 1.73 \mathrm{~m}^{2}$ GFR range revealed a slight positive mean bias of $4.20 \%$ (95\% CI: $0.69-7.70 \%$ ). In the $<60 \mathrm{~mL} / \mathrm{min} /$ $1.73 \mathrm{~m}^{2}$ GFR range, the mean relative bias was $-4.82 \%$, but 0 was included in the $95 \%$ CI ( -25.17 to $15.93 \%)$. A similar observation was made by Salvador et al. [24]. In the latter study, the capillary dried blood spot method overestimated the venous GFR to some extent in the $\geq 60$ $\mathrm{mL} / \mathrm{min} / 1.73 \mathrm{~m}^{2} \mathrm{GFR}$ range. Although in the present study an acceptable mean relative bias was observed in the lower GFR range, only $50 \%$ of the VAMS GFR measurements below $60 \mathrm{~mL} / \mathrm{min} / 1.73 \mathrm{~m}^{2}$ showed an accuracy within $\pm 15 \%$ (P15) of the plasma GFR measurement. In contrast, for GFR values $>60 \mathrm{~mL} / \mathrm{min} / 1.73 \mathrm{~m}^{2}, 94 \%$ of the measurements were within the $\pm 15 \%$ interval (Table 4 ). In this study, primarily neonates demonstrated a GFR $<60 \mathrm{~mL} / \mathrm{min} / 1.73 \mathrm{~m}^{2}$. A possible solution could be the inclusion of a later time point sample. In this way small deviations in calculated plasma VAMS concentrations compared to the plasma concentrations using the conventional method will influence to a lesser extent the slope of the plasma concentration-time profile. Consequently, the slope of the plasma concentration-time profile will be more accurately estimated. This is of major importance, since the slope determines the accurate estimation of the $\mathrm{AUC}_{0 \rightarrow \text { inf. After all, the }}$ $\mathrm{AUC}_{0 \rightarrow \text { inf }}$ is included in the calculations of the iohexol clearance and thus GFR. Consequently, the estimation of the slope determines indirectly the accurate estimation of the GFR. For patients with a severely reduced renal function, sampling up to $24 \mathrm{~h}$ after administration might be recommended. Previously, Ebert et al. demonstrated in older adults 


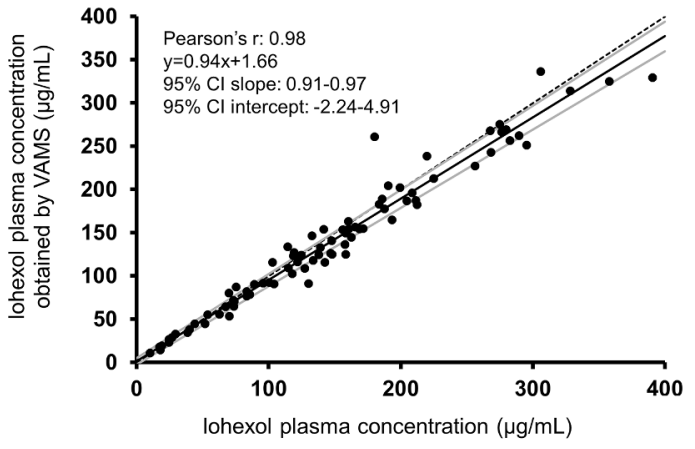

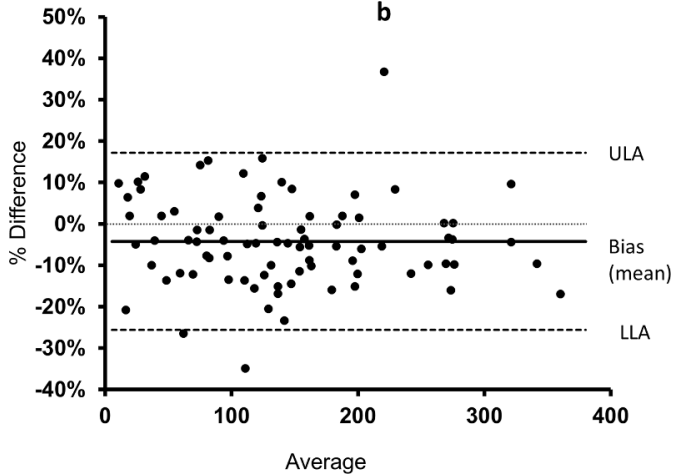

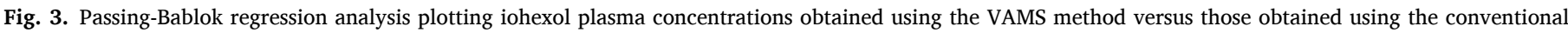

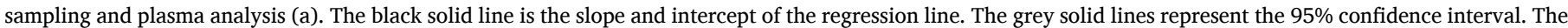

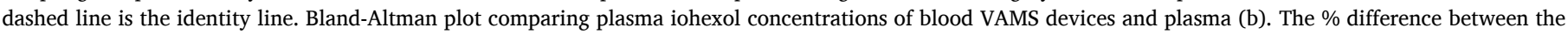

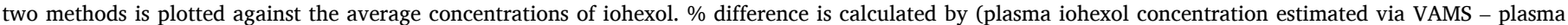

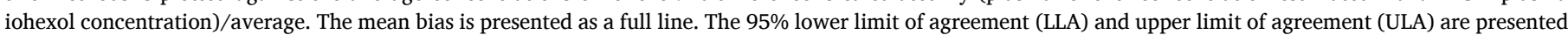
by broken lines.

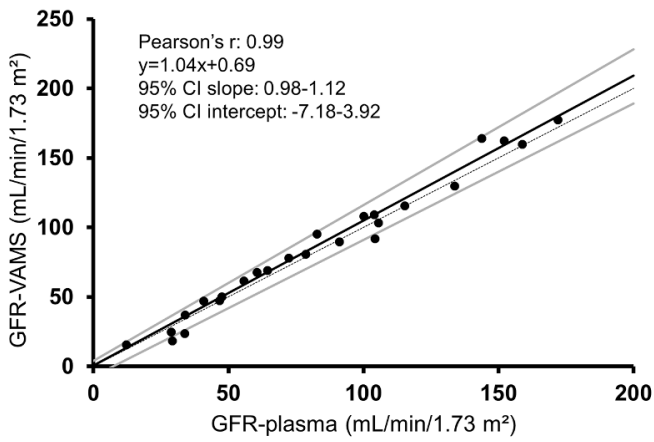

b

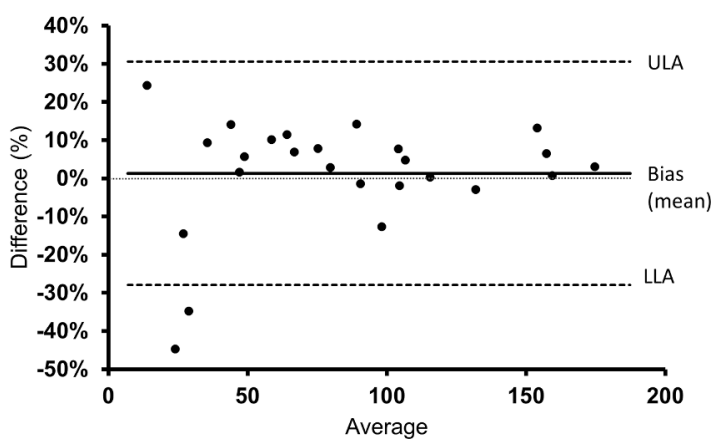

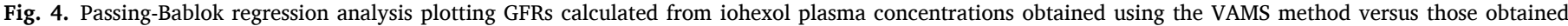

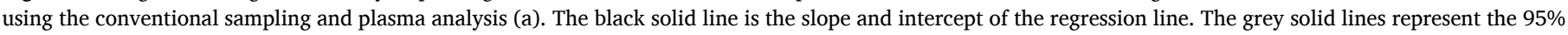

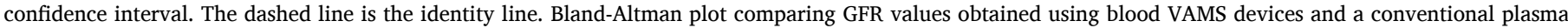

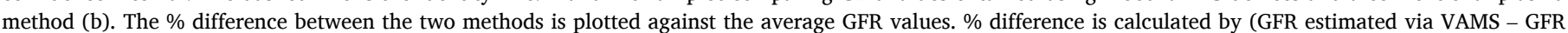

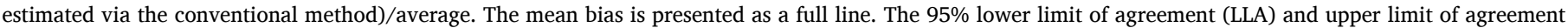
(ULA) are presented by broken lines.

Table 4

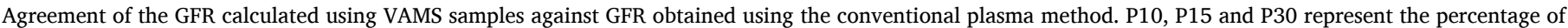

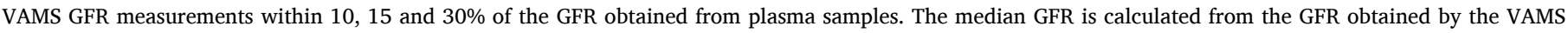
technique.

\begin{tabular}{|c|c|c|c|c|c|c|c|c|c|c|c|}
\hline & $\begin{array}{l}\text { Median GFR } \\
\text { (range) }(\mathrm{mL} / \\
\left.\min / 1.73 \mathrm{~m}^{2}\right)\end{array}$ & $\mathbf{n}$ & $\begin{array}{l}\text { Mean absolute } \\
\text { bias }(\mathrm{mL} / \mathrm{min} / \\
\left.1.73 \mathrm{~m}^{2}\right)\end{array}$ & $\begin{array}{l}95 \% \text { CI bias } \\
(\mathrm{mL} / \mathrm{min} / \\
\left.1.73 \mathrm{~m}^{2}\right)\end{array}$ & $\begin{array}{l}95 \% \operatorname{LoA}(\mathrm{mL} / \\
\left.\mathrm{min} / 1.73 \mathrm{~m}^{2}\right)\end{array}$ & $\begin{array}{l}\text { Mean } \\
\text { relative } \\
\text { bias (\%) }\end{array}$ & $\begin{array}{l}\text { 95\% CI bias } \\
(\%)\end{array}$ & 95\% LoA(\%) & $\begin{array}{l}\text { P10 } \\
(\%)\end{array}$ & $\begin{array}{l}\text { P15 } \\
\text { (\%) }\end{array}$ & $\begin{array}{l}\text { P30 } \\
\text { (\%) }\end{array}$ \\
\hline All & $80.8(15.6-177.4)$ & 25 & 2.49 & $-0.51-5.50$ & $-11.80-16.78$ & 1.31 & $-4.84-7.47$ & $-27.91-30.53$ & 60 & 80 & 96 \\
\hline $\begin{array}{c}\text { GFR < } \\
60 \mathrm{~mL} / \\
\mathrm{min} / \\
1.73 \\
\mathrm{~m}^{2}\end{array}$ & $31.0(15.6-50.2)$ & 8 & -1.01 & $-6.39-4.38$ & $-13.63-11.62$ & -4.82 & $-25.17-15.93$ & $-52.54-42.89$ & 38 & 50 & 88 \\
\hline $\begin{array}{c}\text { GFR }> \\
60 \mathrm{~mL} / \\
\mathrm{min} / \\
1.73 \\
\mathrm{~m}^{2}\end{array}$ & $\begin{array}{l}103.5 \\
(61.5-177.4)\end{array}$ & 17 & 4.14 & $0.41-7.87$ & $-10.07-18.35$ & 4.20 & $0.69-7.70$ & $-9.16-17.56$ & 71 & 94 & 100 \\
\hline
\end{tabular}

$\mathrm{CI}=$ Confidence Interval, LoA $=$ Limits of Agreement.

with chronic kidney disease that measurement of iohexol clearance up to $5 \mathrm{~h}$ leads to a clinically relevant overestimation of GFR compared with 24-h measurement [56].

Since VAMS were generated using whole blood obtained through a catheter and not by conventional finger prick, the most important advantage of the VAMS technique in this study was undoubtedly the limited sampling volume. Because of this limited volume, repeated measurement of the GFR using iohexol is possible in the studied patients. Repeated measurements of the GFR are especially valuable in the critically ill paediatric patient population, since it allows detection of acute 
fluctuations in renal function. Consequently, clinicians are more able to respond appropriately on the presence/evolution of augmented renal clearance or acute kidney injury. In patients without catheter, the use of VAMS microsampling offers, besides the limited sampling volume, some other advantages. First, microsamples are more easily obtained by fingerprick than by conventional blood sampling (phlebotomy). Consequently, microsampling dramatically improves feasibility and reduces cost. In addition, a fingerprick may be less intimidating for children than phlebotomy. Also for nonhospitalized patients, the use of microsampling can be favourable. More specifically, after injection of iohexol by a general practitioner, the patient does not need to remain in-clinic for several hours after administration of the marker in order to collect serial blood samples. Self-collection of VAMS can be performed by the patient (or parent) himself at predefined time points. Subsequently, the dried microsamples can be sent back to the laboratory for analysis. Notwithstanding these advantages, it is important to recognize that collection of VAMS samples requires a high level of training, Therefore, the application of microsampling by the patient (or parent) himself might be only beneficial in patients where repetitive measurements of the GFR are required.

It is important to recognize that this research had limitations. First, it was decided to prepare the VAMS samples by dipping the outer part of the absorbent VAMS tip in arterial or venous blood drawn with a syringe, and not in blood obtained by a conventional fingerprick. Since this study was conducted in paediatric ICU patients, access via a catheter was always available. Hence, via this collection method no extra prick events were mandatory, which is preferable in this young and vulnerable population. Consequently this study does not account for variations due to sampling capillary blood via finger prick. Nevertheless, previous studies demonstrated comparable iohexol results by finger prick and conventional venous blood sampling $[25,26]$. Secondly, our study was performed in a relative small and very heterogeneous population. Further research is necessary to confirm the suitability of the VAMS technology as alternative sampling strategy in a large-scale, multi-centre study, including all age categories, with both (supra-) normal and decreased renal function. Before such a large scale study can be performed, the selection of optimal sampling protocols seems necessary.

\section{Conclusion}

A new, fully validated analytical method was developed for the quantification of iohexol in VAMS samples. This simple and costeffective method fulfilled all pre-set validation acceptance criteria. The haematocrit effect on recovery of iohexol in VAMS was limited, since iohexol could be accurately quantified within a haematocrit range of $20-60 \%$. Furthermore, long-term stability of iohexol in VAMS was demonstrated at $-20^{\circ} \mathrm{C}, 2-8{ }^{\circ} \mathrm{C}$ and room temperature. Iohexol plasma concentrations, obtained by a conventional plasma collection method, and estimated plasma concentrations, obtained from VAMS, demonstrated a good correlation and an acceptable bias. Hence, GFR calculated from VAMS and the conventional plasma method also presented good correlation over the tested GFR range. The agreement between the two methods was satisfactory for GFR values $>60 \mathrm{~mL} / \mathrm{min} / 1.73 \mathrm{~m}^{2}$. Nevertheless, for GFR values $<60 \mathrm{~mL} / \mathrm{min} / 1.73 \mathrm{~m}^{2}$ the agreement was lower.

\section{Funding}

This study was supported by the Special Research Fund of Ghent University (grant number BOF16/DOC/285). Pieter De Cock would like to acknowledge funding from Early Career Award, Trasher Research Fund and Clinical Research Fund (Ghent University Hospital).

\section{Ethics approval}

This study was approved by the Ethics Committee of Ghent
University Hospital (2018/0170).

\section{Consent to participate}

Informed consent was provided by the parents or their legal representative.

\section{Authors contributions}

Laura Dhondt, Pieter De Cock and Evelyn Dhont contributed conception and design of the study. Evelyn Dhont performed the sample collection. Laura Dhondt performed the bioanalytical, pharmacokinetic and statistical analysis and drafted the manuscript. Mathias Devreese aided in the pharmacokinetic analysis. All authors contributed to manuscript revision, read and approved the submitted version.

\section{CRediT authorship contribution statement}

Laura Dhondt: Conceptualization, Methodology, Validation, Investigation, Formal analysis, Writing - original draft, Visualization. Siska Croubels: Supervision, Writing - review \& editing. Pieter De Cock: Conceptualization, Methodology, Writing - review \& editing. Evelyn Dhont: Conceptualization, Methodology, Resources, Writing - review \& editing. Siegrid De Baere: Writing - review \& editing. Peter De Paepe: Writing - review \& editing. Mathias Devreese: Supervision, Writing review \& editing.

\section{Declaration of Competing Interest}

The authors declare that they have no known competing financial interests or personal relationships that could have appeared to influence the work reported in this paper.

\section{Acknowledgements}

The help of Jelle Lambrecht during the sample analyses was gratefully appreciated. The Laboratory of Pharmacology and Toxicology is part of the Ghent University expertise centre MSsmall.

\section{Appendix A. Supplementary data}

Supplementary data to this article can be found online at https://doi. org/10.1016/j.jchromb.2021.122623.

\section{References}

[1] L.A. Stevens, A.S. Levey, Measured GFR as a confirmatory test for estimated GFR, J. Am. Soc. Nephrol. 20 (11) (2009) 2305-2313.

[2] H. Smith, Comparative physiology of the kidney. The kidney: structure and function in health and disease, Oxford University Press, New York, 1954.

[3] L. Wesson, Physiology of the human kidney, Grune \& Stratton, New York, 1969.

[4] L.A. Stevens, A.S. Levey, Measurement of kidney function, Med. Clin. North Am. 89 (3) (2005) 457-473.

[5] P. Bjornstad, A.B. Karger, D.M. Maahs, Measured GFR in routine clinical practiceThe promise of dried blood spots, Adv. Chronic Kidney Dis. 25 (1) (2018) 76-83.

[6] E.J. Lamb, P.E. Stevens, Estimating and measuring glomerular filtration rate: methods of measurement and markers for estimation, Curr. Opin. Nephrol. Hypertens. 23 (3) (2014) 258-266.

[7] P. Delanaye, N. Ebert, T. Melsom, et al., Iohexol plasma clearance for measuring glomerular filtration rate in clinical practice and research: a review. Part 1: How to measure glomerular filtration rate with iohexol? Clin. Kidney J. 9 (5) (2016) 682-699.

[8] K. Skinnemoen, Physicochemical properties and degree of protein binding of iopentol, Acta Radiol. Suppl. 370 (1987) 33-36.

[9] E. Krutzen, S.E. Back, I. Nilssonehle, et al., Plasma clearance of a new contrast agent, iohexol - A method for the assessment of glomerular filtration rate, J. Lab. Clin. Med. 104 (6) (1984) 955-961.

[10] A. Arvidsson, A. Hedman, Plasma and renal clearance of iohexol - A study on the reproducibility of a method for the glomerular filtration rate, Scand. J. Clin. Lab. Invest. 50 (7) (1990) 757-761.

[11] B. Olsson, A. Aulie, K. Sveen, et al., Human pharmacokinetics of iohexol. A new non-ionic contrast-medium, Invest. Radiol. 18 (2) (1983) 177-182. 
[12] F. Carrara, F. Gaspari, GFR measured by iohexol: the best choice from a laboratory perspective, J. Lab Precis. Med. (2018) 3.

[13] F.B. Vicente, G.K. Vespa, F. Carrara, et al., Determination of iohexol in human serum by a semi-automated liquid chromatography tandem mass spectrometry method, Clin. Biochem. 48 (10-11) (2015) 679-685.

[14] T.M. Annesley, L.T. Clayton, Ultraperformance liquid chromatography-tandem mass spectrometry assay for iohexol in human serum, Clin. Chem. 55 (6) (2009) 1196-1202.

[15] M.C. Denis, K. Venne, D. Lesiege, et al., Development and evaluation of a liquid chromatography-mass spectrometry assay and its application for the assessment of renal function, J. Chromatogr. A 1189 (1-2) (2008) 410-416.

[16] D. Farthing, D.A. Sica, I. Fakhry, et al., Simple HPLC-UV method for determination of iohexol, iothalamate, p-aminohippuric acid and n-acetyl-p-aminohippuric acid in human plasma and urine with ERPF, GFR and ERPF/GFR ratio determination using colorimetric analysis, J. Chromatogr. B 826 (1-2) (2005) 267-272.

[17] R.S. Soman, H. Zahir, F. Akhlaghi, Development and validation of an HPLC-UV method for determination of iohexol in human plasma, J. Chromatogr. B 816 (1-2) (2005) 339-343.

[18] Z.K. Shihabi, M.S. Constantinescu, Iohexol in serum determined by capillary electrophoresis, Clin. Chem. 38 (10) (1992) 2117-2120.

[19] M.V. Rocco, V.M. Buckalew, L.C. Moore, et al., Capillary electrophoresis for the determination of glomerular filtration rate using nonradioactive iohexol, Am. J. Kidney Dis. 28 (2) (1996) 173-177.

[20] G. Stake, E. Monn, K. Rootwelt, et al., Glomerular filtration rate estimated by X-ray fluorescence technique in children: Comparison between the plasma disappearance of 99Tcm-DTPA and iohexol after urography, Scand. J. Clin. Lab. Invest. 50 (2) (1990) 161-167.

[21] G. Nys, M.G. Kok, A.-C. Servais, et al., Beyond dried blood spot: current microsampling techniques in the context of biomedical applications, TrAC, Trends Anal. Chem. 97 (2017) 326-332.

[22] M.M. Mafham, I. Niculescu-Duvaz, J. Barron, et al., A practical method of measuring glomerular filtration rate by iohexol clearance using dried capillary blood spots, Nephron Clin. Pract. 106 (3) (2007) c104-c112.

[23] I. Niculescu-Duvaz, L. D'mello, Z. Maan, et al., Development of an outpatient finger-prick glomerular filtration rate procedure suitable for epidemiological studies, Kidney Int. 69 (7) (2006) 1272-1275.

[24] C.L. Salvador, C. Tondel, L. Morkrid, et al., Glomerular filtration rate measured by iohexol clearance: A comparison of venous samples and capillary blood spots, Scand. J. Clin. Lab. Invest. 75 (8) (2015) 710-716.

[25] A. Staples, C. Wong, G.J. Schwartz, Iohexol-measured glomerular filtration rate in children and adolescents with chronic kidney disease: a pilot study comparing venous and finger stick methods, Pediatric Nephrol. 34 (3) (2019) 459-464.

[26] S. Luis-Lima, F. Gaspari, N. Negrin-Mena, et al., Iohexol plasma clearance simplified by dried blood spot testing, Nephrol. Dial. Transplant. 33 (9) (2018) 1597-1603.

[27] T. Dorofaeff, R.M. Bandini, J. Lipman, et al., Uncertainty in antibiotic dosing in critically ill neonate and pediatric patients: Can microsampling provide the answers? Clin. Ther. 38 (9) (2016) 1961-1975.

[28] B.U.W. Lei, T.W. Prow, A review of microsampling techniques and their social impact, Biomed. Microdevices 21 (4) (2019).

[29] A.J. Lawson, L. Bernstone, S.K. Hall, Newborn screening blood spot analysis in the UK: influence of spot size, punch location and haematocrit, J. Med. Screen. 23 (17-16) (2016).

[30] M.G. Kok, M. Fillet, Volumetric absorptive microsampling: current advances and applications, J. Pharm. Biomed. Anal. 147 (2018) 288-296.

[31] V. Ion, C. Legoff, E. Cavalier, et al., Determination of iohexol by capillary blood microsampling and UHPLC-MS/MS, J. Pharm. Anal. 9 (4) (2019) 259-265.

[32] European Medicines Agency. Guideline on bioanalytical method validation, 2011.

[33] J. Knecht, G. Stork, Prozentuales und logarithmisches verfahren zur berechnung von eichkurven, Z. Anal. Chem 270 (1974) 97-98.

[34] Committee for Medicinal Products for Veterinary Use. VICH Topic GL49: Studies to Evaluate the Metabolism and Residue Kinetics of Veterinary Drugs in Food Producing Animals: Validation of Analytical Methods Used in Residue Depletion Studies, 2015.
[35] N. Spooner, K.D. Anderson, J. Siple, et al., Microsampling: considerations for its use in pharmaceutical drug discovery and development, Bioanalysis 11 (10) (2019) 1015-1038.

[36] S. Capiau, H. Veenhof, R.A. Koster, et al., Official international association for therapeutic drug monitoring and clinical toxicology guideline: Development and validation of dried blood spot-based methods for therapeutic drug monitoring, Ther. Drug Monit. 41 (4) (2019) 409-430.

[37] B. Matuszewski, M. Constanzer, C. Chavez-Eng, Strategies for the assessment of matrix effect in quantitative bioanalytical methods based on HPLC- MS/MS, Anal. Chem. 75 (13) (2003) 3019-3030.

[38] D. Dubois, E.F. Dubois, A formula to estimate the approximate surface area if height and weight be known, Arch. Intern. Med. 17 (1916) 863-871.

[39] E. Krutzen, S. Bäck, P. Nilsson-Ehle, Determination of glomerular filtration rate using iohexol clearance and capillary sampling, Scand. J. Clin. Lab. Invest. 50 (3) (1990) 279-283.

[40] L. Dhondt, S. Croubels, P. De Cock, et al., Development and validation of an ultrahigh performance liquid chromatography-tandem mass spectrometry method for the simultaneous determination of iohexol, p-aminohippuric acid and creatinine in porcine and broiler chicken plasma, J. Chromatogr. B 1117 (2019) 77-85.

[41] F. Gaspari, N. Perico, P. Ruggenenti, et al., Plasma clearance of nonradioactive iohexol as a measure of glomerular filtration rate, J. Am. Soc. Nephrol. 6 (2) (1995) $257-263$.

[42] C. Tøndel, B. Bolann, C.L. Salvador, et al., Iohexol plasma clearance in children: validation of multiple formulas and two-point sampling times, Pediatric Nephrol. 32 (2) (2017) 311-320.

[43] D.J. Schmit, L.J. Carroll, J.H. Eckfeldt, et al., Verification of separate measurement procedures where analytical determinations influence the clinical interpretation of GFR: Iohexol quantitation by HPLC and LC-MS/MS, Clin. Biochem. 67 (2019) 16-23.

[44] W. Zhou, S. Yang, P.G. Wang, Matrix effects and application of matrix effect factor, Bioanalysis 9 (23) (2017) 1839-1844.

[45] H. Mei, Y. Hsieh, C. Nardo, et al., Investigation of matrix effects in bioanalytical high-performance liquid chromatography/tandem mass spectrometric assays: application to drug discovery, Rapid Commun. Mass Spectrom. 17 (1) (2003) 97-103.

[46] A.D. Cornet, E.G. Smit, A. Beishuizen, et al., The role of heparin and allied compounds in the treatment of sepsis, Thromb. Haemost. 98 (09) (2007) 579-586.

[47] S.L. Parker, J.A. Roberts, J. Lipman, et al., Quantitative bioanalytical validation of fosfomycin in human whole blood with volumetric absorptive microsampling, Bioanalysis 7 (19) (2015) 2585-2595.

[48] Y. Mano, K. Kita, K. Kusano, Hematocrit-independent recovery is a key for bioanalysis using volumetric absorptive microsampling devices, Mitra(TM), Bioanalysis 7 (15) (2015) 1821-1829.

[49] K. Adeli, J.E. Raizman, Y. Chen, et al., Complex biological profile of hematologic markers across pediatric, adult, and geriatric ages: establishment of robust pediatric and adult reference intervals on the basis of the Canadian Health Measures Survey, Clin. Chem. 61 (8) (2015) 1075-1086.

[50] R. Hinchliffe, G. Bellamy, F. Bell, et al., Reference intervals for red cell variables and platelet counts in infants at 2, 5 and 13 months of age: a cohort study, J. Clin. Pathol. 66 (11) (2013) 962-966.

[51] W.L. Mandala, E.N. Gondwe, J.M. MacLennan, et al., Age-and sex-related changes in hematological parameters in healthy Malawians, J. Blood Med. 8 (2017) 123.

[52] L. Delahaye, E. Dhont, P. De Cock, et al., Volumetric absorptive microsampling as an alternative sampling strategy for the determination of paracetamol in blood and cerebrospinal fluid, Anal. Bioanal. Chem. 411 (1) (2019) 181-191.

[53] Food and Drug Administration (FDA) Guidance for Industry: Bioanalytical Method Validation, 2018.

[54] C.R. Gleason, Q.C. Ji, E.R. Wickremsinhe, Evaluation of correlation between bioanalytical methods, Bioanalysis 12 (6) (2020) 419-426.

[55] D. Giavarina, Understanding bland altman analysis, Biochem. Med.: Biochem. Med. 25 (2) (2015) 141-151.

[56] N. Ebert, A. Loesment, P. Martus, et al., Iohexol plasma clearance measurement in older adults with chronic kidney disease-sampling time matters, Nephrol. Dial. Transplant. 30 (8) (2015) 1307-1314. 